\title{
A Kind of Neither Keynesian Nor Neoclassical Model (2): The Business Cycle
}

\author{
Ming'an Zhan', Zhan Zhan² \\ ${ }^{1}$ Yunnan University, Kunming, China \\ ${ }^{2}$ Westa College, Southwest University, Chongqing, China \\ Email:1792481782@qq.com, blacktea1313ryan@hotmail.com
}

How to cite this paper: Zhan, M.A. and Zhan, Z. (2016) A Kind of Neither Keynesian Nor Neoclassical Model (2): The Business Cycle. Open Access Library Journal, 3: e3215.

http://dx.doi.org/10.4236/oalib.1103215

Received: November 9, 2016

Accepted: December 19, 2016

Published: December 23, 2016

Copyright $\odot 2016$ by authors and Open Access Library Inc.

This work is licensed under the Creative Commons Attribution International

License (CC BY 4.0).

http://creativecommons.org/licenses/by/4.0/

\begin{abstract}
The Cobb-Douglas function not only leads to a long-term relationship between the rate of output change and the interest rate, but also analyzes why they fluctuate in the short-term. This paper first divides the fluctuation cycle of the interest rate in the statistical data of the past 45 years by using the mathematical phase diagram method, and draws the phase diagram of the rate of output change on the interest rate according to the cycle equation of output. From this phase diagram, we explain the reason that the phase difference between the interest rate and the rate of output change in the fluctuation. Then, according to the optimal relation between $L$ and $K$ in the Cobb-Douglas function, we further derive the employment equation and its relation to the real interest rate and the rate of real output change, and verify the theoretical speculation with statistical data. Finally, it is concluded that the business cycle is a kind of endogenous production phenomenon.
\end{abstract}

\section{Subject Areas}

Economics

Keywords

Cobb-Douglas Function, Business Cycles, Phase Diagram, Unemployment Rate

\section{Introduction}

Neoclassic theories believe that the surplus of output and unemployment would be cleaned out during competition and equilibrium is the normality of the economic system, therefore the fluctuation of macroeconomic variables is generated by external factors. The "Real Business Cycle Model" (RBC) arisen during 1980s considers the total factor productivity or the random perturbations of technology determine fluctuation of 
other variables [1] [2] [3]. However, other economists [4] [5] gave this theory some questions: according to $\mathrm{RBC}$, if the economic boom was generated by the technological progress, then the economic recession should blame on technological setbacks. Nevertheless, what are reasons of technological setbacks? Moreover, if we use other methods to measure the technological impact, it can be a factor that eliminates business cycles [6] [7] [8] [9] [10].

Hayek said: "the incorporation of cyclical phenomena into the system of economic equilibrium theory, which they are on apparent contradiction." [11]. Since the interaction of the total supply and total demand leads to convergence rather than divergence, the convergence would flatten the fluctuation ever if there were external impacts assumed by the RBC theory. Perhaps the biggest problem with macroeconomics is explaining business cycles.

\section{The Business Cycle Equation and Divisions}

From the Cobb-Douglas function $Y=A K^{\alpha} L^{\beta}$, we deduce the marginal revenue of the capital Kin the paper "A kind of neither Keynesian nor neoclassical model (1): fundamental equation" [12]:

$$
M P K=\frac{\partial Y}{\partial K}=\frac{\alpha Y}{K}
$$

In a competitive market, assuming that the marginal cost of using $K$ is determined by the market interest rate $r$, the optimal allocation condition for $K$ in production is $M P K=r$, then

$$
r=\frac{\alpha Y}{K} \text { or } Y=\frac{r K}{\alpha},
$$

differential on both sides of the equation, so:

$$
\frac{\mathrm{d} Y}{Y}=\frac{\mathrm{d} K}{K}+\frac{\mathrm{d} r}{r}-\frac{\mathrm{d} \alpha}{\alpha},
$$

according to the basic equation, $\Delta K=r K$

$$
\frac{\mathrm{d} K}{K}=\lim _{\Delta K \rightarrow 0} \frac{\Delta K}{K}=\lim _{\Delta K \rightarrow 0} \frac{r K}{K}=r,
$$

so Equation (3) can be rewritten as:

$$
\frac{\mathrm{d} Y}{Y}=r+\frac{\mathrm{d} r}{r}-\frac{\mathrm{d} \alpha}{\alpha}
$$

When $r$ and $\alpha$ are constant, $\mathrm{d} r / r=0, \mathrm{~d} \alpha / \alpha=0, \mathrm{~d} Y / Y=r$. This is the macroeconomic basic equation. Equation (5) contains more information than the fundamental equation $\mathrm{d} Y / Y=r$. It can be used to analyze the relationship of short-term fluctuations between $\mathrm{d} Y / Y$ and $r$.

In $Y=A K^{\alpha} L^{\beta}$, the income distribution parameter $\alpha$ mainly affects the long-term growth (We will analyze this problem in another paper "The economic growth"), assuming $\mathrm{d} \alpha / \alpha=0$, then Equation (5) can be simplified as: 


$$
\frac{\mathrm{d} Y}{Y}=r+\frac{\mathrm{d} r}{r}
$$

This is the business cycle equation. The change of $\mathrm{d} Y / Y$ in short term depends on changes of $r$ and $\mathrm{d} r / r$. The interaction between $r$ and $\mathrm{d} r / r$ might leads to endless fluctuation of $\mathrm{d} Y / Y$.

By $r=M P K$ we can see that $r$ reflects the production efficiency in the use of $K$. The periodic fluctuation in the statistical data can be described by the phase diagram in the mathematical model. Using $r$ as the horizontal axis and $\mathrm{d} r / r$ as the vertical axis, we can plot the phase diagram of $r \sim \mathrm{d} r / r$.

As shown in Figure 1(a), since $\mathrm{d} r / r=0, \mathrm{~d} r=0$ at points A and C, so $r$ should not continue to change. However, since $\mathrm{d} r=0, \mathrm{~d}(\mathrm{~d} r / r) / \mathrm{d} r \neq 0$, the point $\mathrm{A}$ or $\mathrm{C}$ is not a stable point on the Phase diagram $r \sim \mathrm{d} r / r$.

At point $\mathrm{B}$ or $\mathrm{D}, \mathrm{d} r / r \neq 0$, so $r$ will continue to change. Therefore, once $r$ moves up, the phase diagram $r \sim \mathrm{d} r / r$ will endogenously change and make $\mathrm{d} Y / Y$ in equation (7) periodically fluctuate. Moreover, we will see that the fluctuations of other variables are directly or indirectly related to the fluctuation of $r$.

According to the phase diagram $r \sim \mathrm{d} r / r$, we can define the characteristic of periodic change of $r$, or be called the three elements of the phase diagram: firstly, point $\mathrm{E}$ in Figure 1(a) is the core of one cycle, and the interest rate that corresponds to point $\mathrm{E}$ is an average value of interest rate of a business cycle ( $\bar{r}$, namely the core interest rate). Secondly, EA or EC is the radius of fluctuation of $r$. Thirdly, the rotation of the phase diagram $r \sim \mathrm{d} r / r$ is clockwise. Theoretically, we can call four phases of $\mathrm{A} \rightarrow \mathrm{B}, \mathrm{B} \rightarrow \mathrm{C}$, $\mathrm{C} \rightarrow \mathrm{D}$ and $\mathrm{D} \rightarrow \mathrm{A}$ in Figure 1 as period of recovery, prosperity, recession and depression.

Based on the phase diagram $r \sim \mathrm{d} r / r$, we can easily draw out the time path of $r$ as Figure 1(b) shows. In reality, since the value of core interest rate $\bar{r}$ might be affected by external factors, such as the technological impact, therefore the location and radius of $r \sim \mathrm{d} r / r$ can be affected also. Therefore the time path of $r$ cannot be a regular path as trigonometric functions.

Figure 2 is a phase diagram $r \sim \mathrm{d} r / r$ based on statistical data of the United States during 1970-2015. Although we can distinguish different cycles by these phase diagrams (as indicated by the dashed line in Figure 2 for two periods of 1972-1976 and 1976-1983, the cycle starting point is always on $r$-axis or the nearest it below), most of them have only two phases of rise and fall, without theoretical four phases of recovery, prosperity, recession and depression. Some of them even rotate counterclockwise, for example, during 1983-1985.

This is caused by the collection of data has longer interval time than the real fluctuation. Take semi-annual data to redraw this diagram, it is the dotted curve rather than the solid curve during 1983-1985. Therefore, 1976-1983 and 1983-1986 are two different cycles. In the same way, 2003-2009 and 2009-2012 are also two cycles in 2003-2012.

According to annual statistical data and the dividing rule showed in Figure 1(a), there are 9 cycles of fluctuation of interest rate during 1970-2015 of the United States: 1972-1976, 1976-1983, 1983-1986, 1986-1993, 1993-1998, 1998-2003, 2003-2009, 20092012, 2012-. According to the present statistical data (10/2016), $r$ in 2016 may not be 


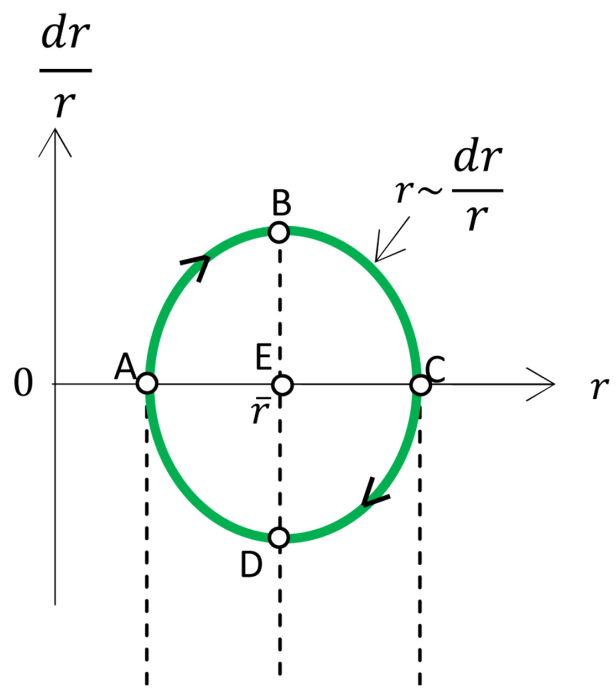

(a)

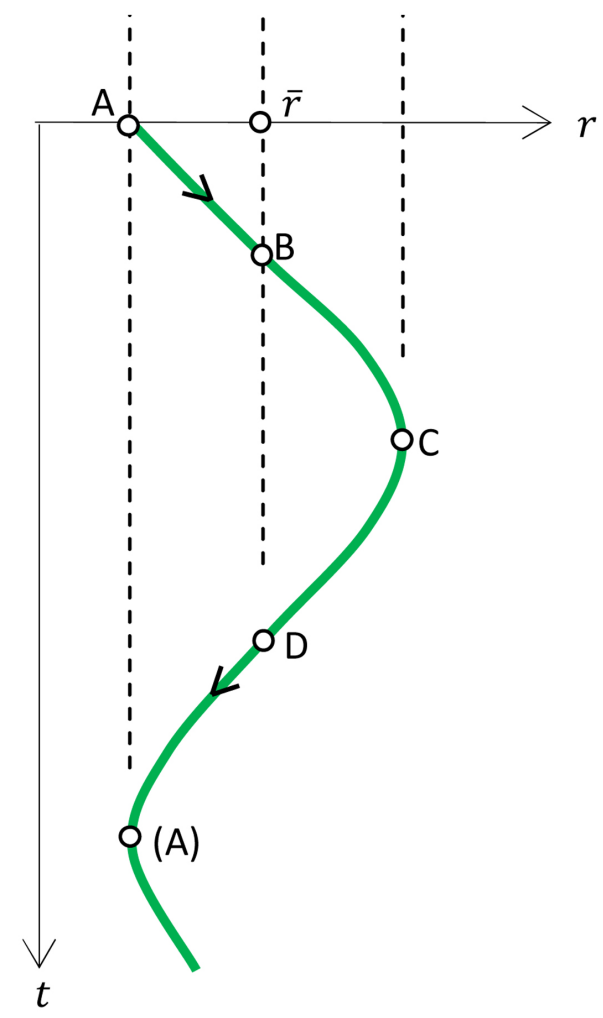

(b)

Figure 1. Mathematical phase diagram about periodic fluctuation of $r$. (a) Phase diagram $r \sim$ $d r / r$. (b) Time path of $r$.

higher than 2015, so we guess 2016 is also in the cycle since 2012. Figure 3 shows the corresponding time path. 


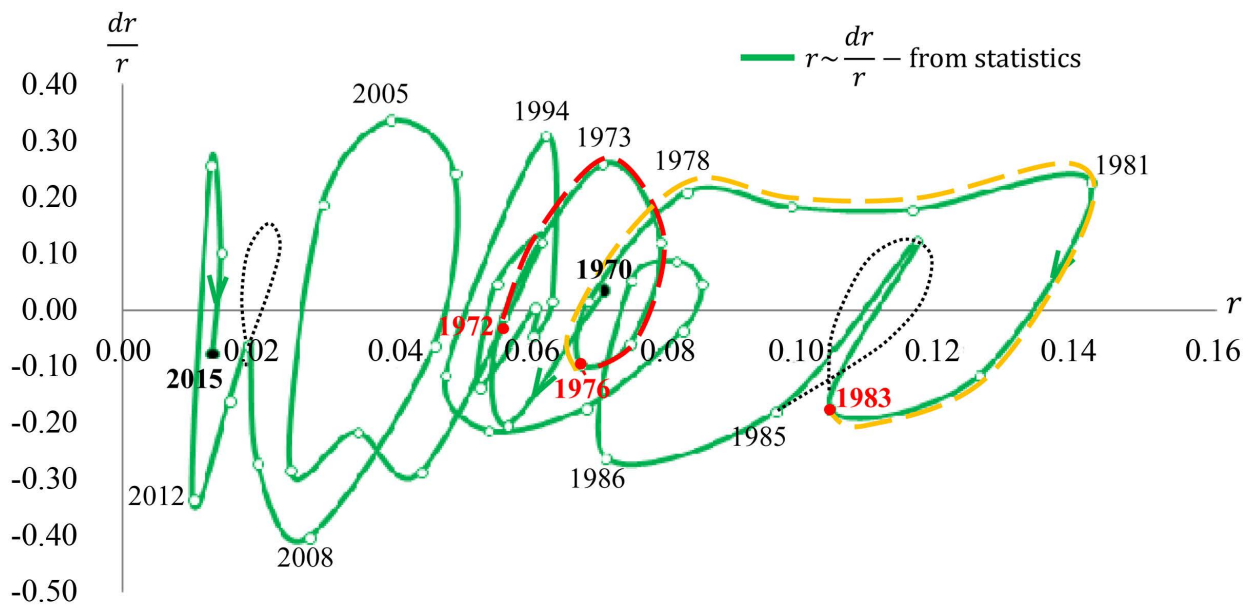

Figure 2. Phase diagram $r \sim d r / r$ based on statistical data. Sources: 1) $d r / r \approx \Delta r / r$. 2) In this figure, $r$ is the nominal, calculated by the simple average of the annualized $r$ of various treasury bonds in the market. The varieties of treasury bonds we collect are 3-months, 6-months, 1-year, 2-year, 5-year, 7-year, 10-year, 20-year and 30-year (Resources: http://www.federalreserve.gov/). Their average is $r$ in the graph. Although statistics of $r$ in some years are incomplete, but we still calculate the average of $r$ based on other varieties. From 1969, there are six varieties of more statistical data, so we will be 1969 as the starting year. 3) Solid curve is generated by Office-Excel automatically. Dotted curve is drawn artificially to show the path when $r$ and $\mathrm{d} r / r$ are semi-annual values.

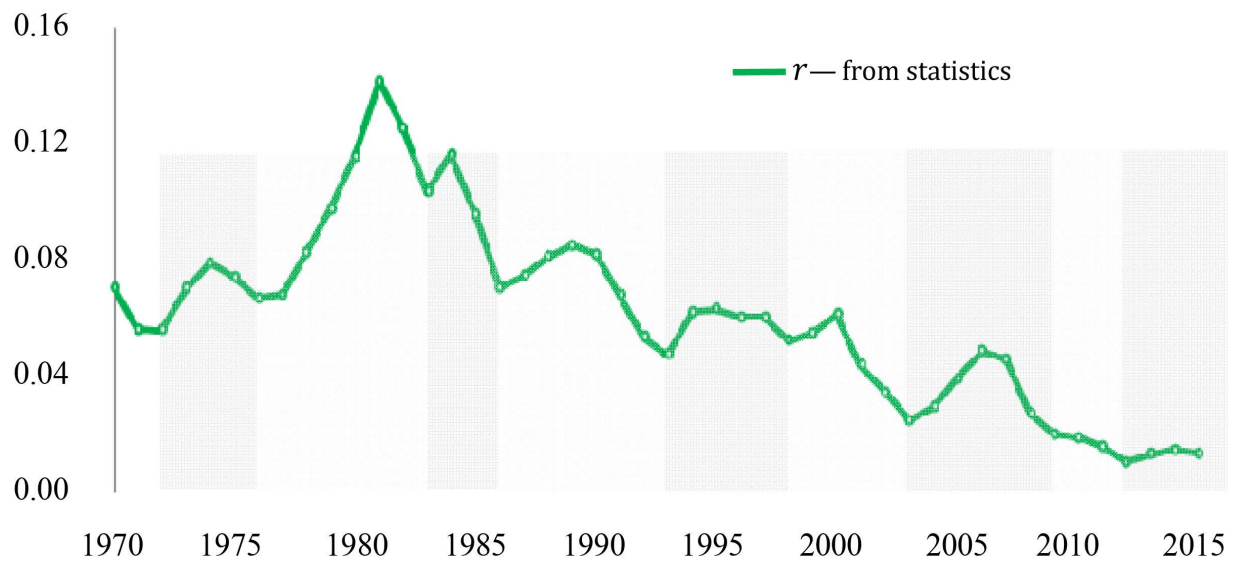

Figure 3. Business cycles divided by the change of $r$. Sources: Light and dark areas show different cycles. Figure 2 shows base of the phase diagram $r \sim \mathrm{d} r / r$. Data of $r$ are the same as Figure 2 .

\section{The Relationship between the Periodicity of $\mathrm{d} Y / Y$ and $r$}

According to Equation (6), the change rate of output $\mathrm{d} Y / Y$ is not only related to $r$ but also to the change rate of interest rate $\mathrm{d} r / r$. Based on the phase diagram $r \sim \mathrm{d} r / r$, we can draw a phase diagram $r \sim \mathrm{d} Y / Y$ as Figure 4 shows. In order to deduce the phase diagram $r \sim \mathrm{d} Y / Y$ from $r \sim \mathrm{d} r / r$, we first draw an auxiliary line which is $\mathrm{d} Y / Y=r$, then fix points $\mathrm{a}, \mathrm{b}, \mathrm{c}, \mathrm{d}$ and e that correspond to points $\mathrm{A}, \mathrm{B}, \mathrm{C}, \mathrm{D}$ and $\mathrm{E}$ on $r \sim \mathrm{d} r / r$ based on $\mathrm{d} Y / Y=r+\mathrm{d} r / r$. 


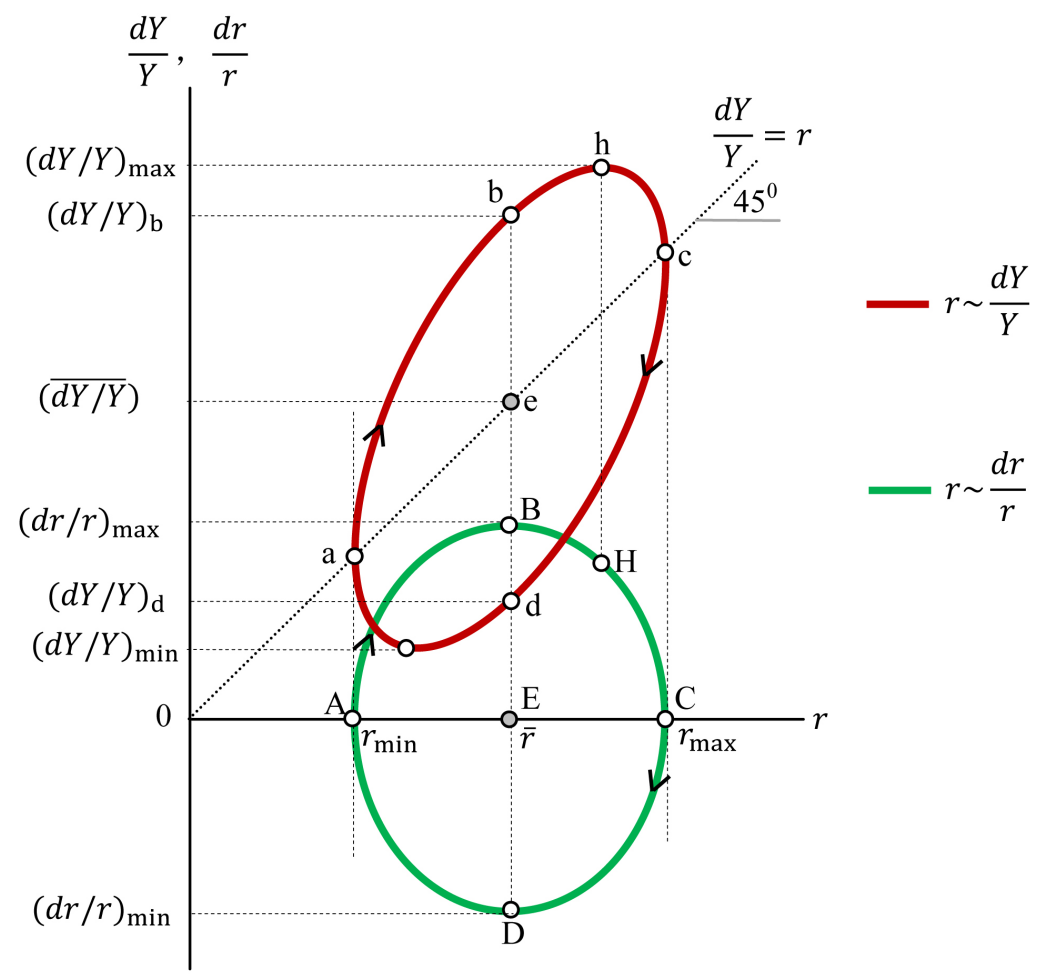

Figure 4. Phase diagram $r \sim \mathrm{d} Y / Y$. Notice: In Figure 4, find a, e, c based on the auxiliary line, and find other points based on phase diagram $r \sim \mathrm{d} r / r$ and equation $\mathrm{d} Y / Y=r+\mathrm{d} r / r$. Since point h on phase diagram $r \sim \mathrm{d} r / r$ is consistent with that on $r \sim \mathrm{d} Y / Y$, therefore $(\mathrm{d} Y / Y)_{\max }$ exists earlier than $r_{\max }$.

Point a and $\mathrm{c}$ on $r \sim \mathrm{d} Y / Y$ are easy to be fixed, since on these two points $\mathrm{d} Y / Y=r$, $\mathrm{d} r / r=0$ (on the phase diagram $\mathrm{d} r / r=0, r$ is the minimum value $r_{\min }$ or the maximum value $\left.r_{\max }\right)$, therefore point a and $\mathrm{c}$ are located on the auxiliary line $\mathrm{d} Y / Y=r$. Points $\mathrm{b}, \mathrm{d}$ and e correspond to the maximum, minimum and average values of $\mathrm{d} r / r$. In that case, $(\mathrm{d} Y / Y)_{\mathrm{b}}=\bar{r}+(\mathrm{d} r / r)_{\max },(\mathrm{d} Y / Y)_{\mathrm{d}}=\bar{r}+(\mathrm{d} r / r)_{\min }$. Similarly, we can determine the other points, connect these points to get the phase diagram $r \sim \mathrm{d} Y / Y$.

Figure 5 shows the time path of $\mathrm{d} Y / Y$ and $r$ according to $r \sim \mathrm{d} Y / Y$. Figure 5(b) and Figure 5(c) are time paths of $\mathrm{d} Y / Y$ and $r$, respectively. In order to compare, we exchange the horizontal and vertical coordinates of Figure 5(b) to get Figure 5(d). By comparing Figure 5(c) and Figure 5(d), there is different of fluctuation of $\mathrm{d} Y / Y$ and $r$. We call it "phase difference".

As shown in Figure 5(c) and Figure 5(d), the time path of $\mathrm{d} Y / Y$ and $r$ are related as: when $r$ increases, $\mathrm{d} Y / Y>r$. From point b to $c$, when $\mathrm{d} Y / Y$ decreases, $r$ keeps increase. Similarly, when $r$ decreases, $\mathrm{d} Y / Y<r$. And from point $\mathrm{d}$ to a, when $\mathrm{d} Y / Y$ starts to increase, $r$ keeps decrease. This shows the peak and trough of $\mathrm{d} Y / Y$ exist earlier than that of $r$. Although the change of $r$ might exist later that of $\mathrm{d} Y / Y$, it does not mean $\mathrm{d} Y / Y$ is the cause of the change of $r$. Contrarily, the change of $\mathrm{d} Y / Y$ is the result of interaction of $r$ and $\mathrm{d} r / r$. 


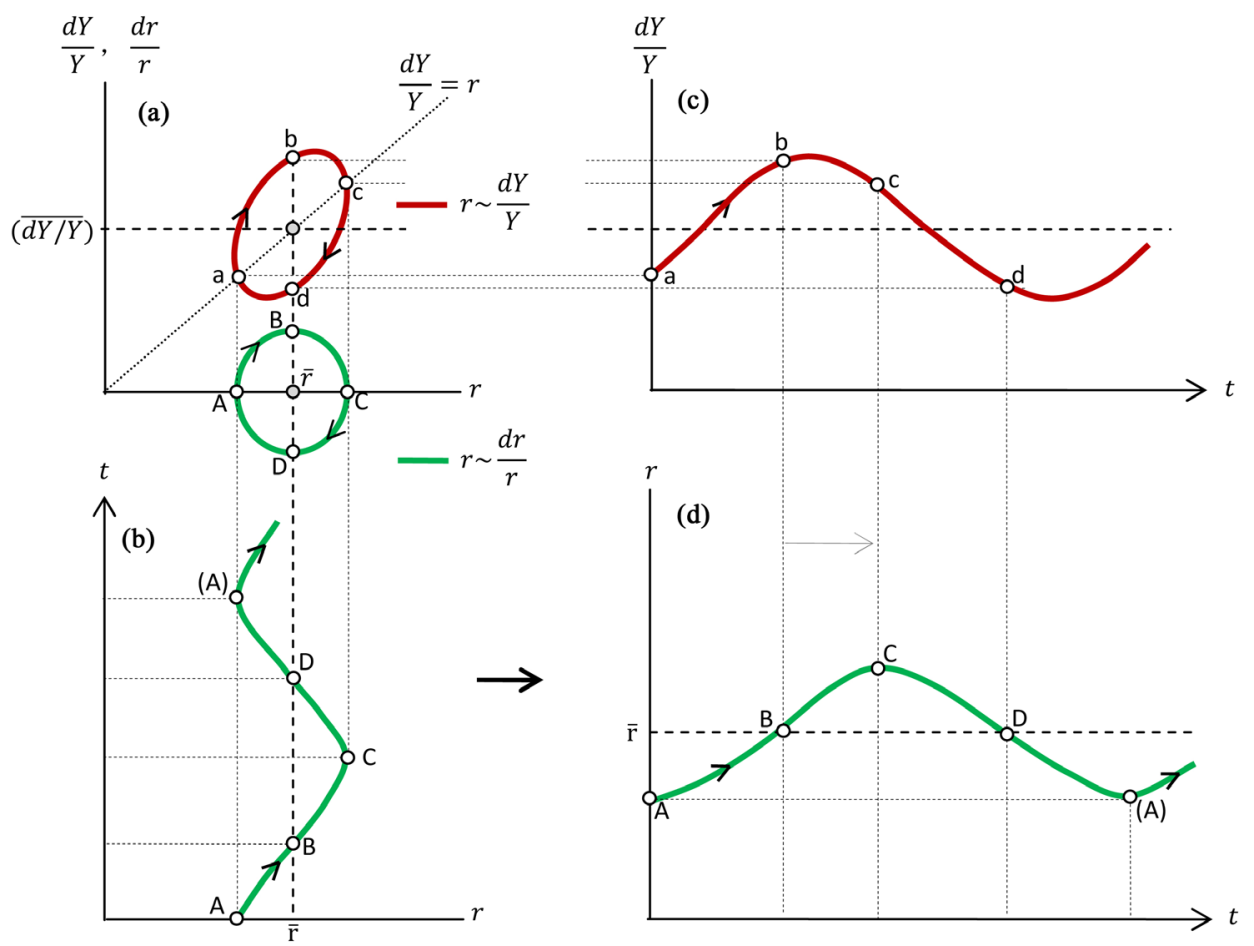

Figure 5. Phase difference of time paths of $\mathrm{d} Y / Y$ and $r$.

In Figure 6, statistical data during 1970-2015 verified the phase difference between time paths of $\mathrm{d} Y / Y$ and $r$. Figure 4 is generated under the assumption of $\mathrm{d} \alpha / \alpha=0$. If $\mathrm{d} \alpha / \alpha \neq 0$, then the auxiliary line $\mathrm{d} Y / Y=r$ should be $\mathrm{d} Y / Y=r-\mathrm{d} \alpha / \alpha$. Therefore when $\mathrm{d} \alpha / \alpha>0$, the phase diagram $r \sim \mathrm{d} Y / Y$ would move downwards or be deflected, vice versa. So the flunctuation of $\mathrm{d} Y / Y$ in real world is much more complex than that of $r$, the peak or trough of $\mathrm{d} Y / Y$ would move forward or backward in the business cycle under the effect of $\mathrm{d} \alpha / \alpha$.

The foundation equation $\mathrm{d} Y / Y=r$ derived assuming that $r$ and $\alpha$ are constant. According to Figure 4, it should be rewritten as $(\overline{\mathrm{d} Y / Y})=\bar{r}$. Although the core rate of output growth $(\overline{\mathrm{d} Y / Y})$ is equal to the core interest rate $\bar{r}$, the value of $\mathrm{d} Y / Y$ and rare not always equal, since them have different phases. Compared to the analysis of total demand and supply, the analysis of phase diagram could give better explanation of relations between short-terms values of macroeconomic variables.

\section{Periodicity of the Unemployment Rate}

Based on algebraic rules, no matter what are the original state of $\alpha$ and $\beta$, there is a dynamic upper limit of Cobb-Douglas function $Y=A K^{\alpha} L^{\beta}$ :

$$
Y=A K^{\alpha} L^{\beta} \leq A \frac{\left(K^{\alpha}\right)^{2}+\left(L^{\beta}\right)^{2}}{2}
$$

Apparently, the condition of $A K^{\alpha} L^{\beta}$ to reach its upper limit is:

$$
L^{\beta}=K^{\alpha}
$$




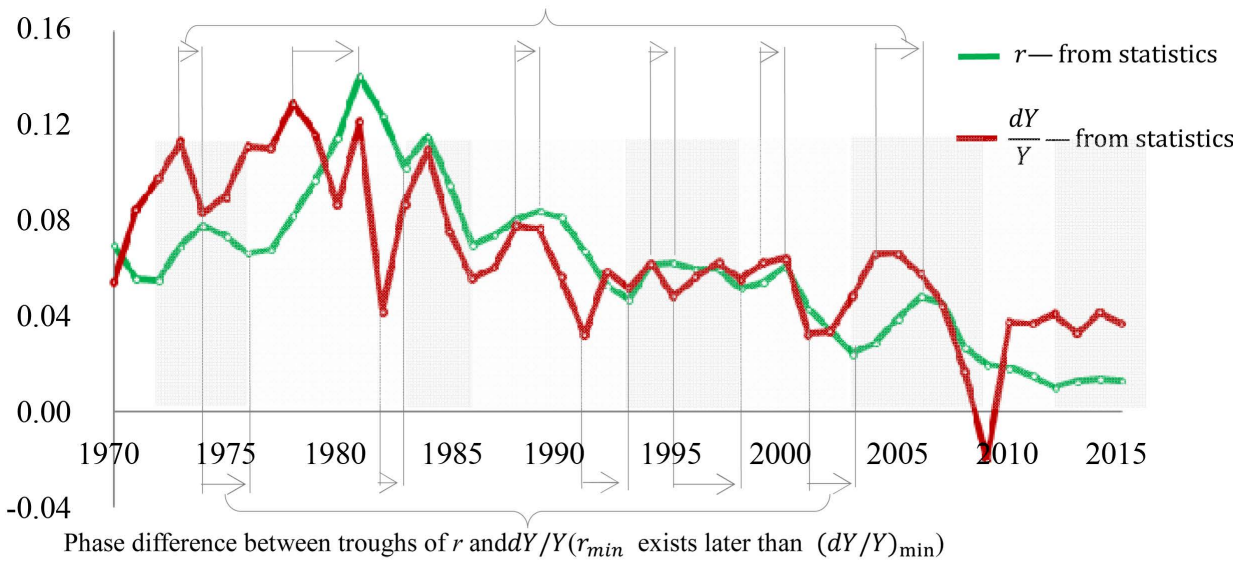

Figure 6. Phase differences between statistical data $\mathrm{d} Y / Y$ and $r$. Sources: 1) $\mathrm{d} Y / Y \approx \Delta Y / Y$, $Y=$ GDP . Data of nominal GDP are from http://www.bea.gov/. 2) Shadowed areas show business cycles as in Figure 3 (the same as hereafter). Data of $r$ are the same as Figure 2.

Equation (8) $L^{\beta}=K^{\alpha}$ not only expresses the endogenous optimization of $L$ and $K$, but also defines the relation between $L$ and $K$. According to economic principles, the amount of production factors used in the system is determined by its marginal revenue. Therefore the amount of $L$ that be used should be closely related to the marginal output of $L(\partial Y / \partial L)$. Based on the marginal equation of $Y=A K^{\alpha} L^{\beta}: \partial Y / \partial L=\beta Y / L$, assume $w=\partial Y / \partial L$, then

$$
w=\frac{\partial Y}{\partial L}=\frac{\beta Y}{L}
$$

since $L^{\beta}=K^{\alpha}, w=\frac{\beta Y}{K^{\frac{\alpha}{\beta}}}, \frac{\mathrm{d} w}{w}=\frac{\mathrm{d}\left(\frac{\beta Y}{K^{\alpha / \beta}}\right)}{\frac{\beta Y}{K^{\alpha / \beta}}}=\frac{\mathrm{d} \beta}{\beta}+\frac{\mathrm{d} Y}{Y}-\left[\frac{\alpha}{\beta} \frac{\mathrm{d} K}{K}+\frac{\alpha}{\beta} \ln K \mathrm{~d}\left(\frac{\alpha}{\beta}\right)\right]$

among them, $\alpha+\beta=1, \mathrm{~d} K / K=r$ ("A kind of neither Keynesian nor neoclassical model (1): The fundamental equation" [12]), Assuming $\alpha$ is a constant, so $\mathrm{d} \beta / \beta=0$ and $\mathrm{d}(\alpha / \beta)=0$, then

$$
\frac{\mathrm{d} w}{w}=\frac{\mathrm{d} Y}{Y}-\frac{\alpha}{\beta} r
$$

In $Y=A K^{\alpha} L^{\beta}, L$ is the monetized human resources, we cannot directly use amount of the employment population $E$ as amount of $L$. There might be relation between $\mathrm{d} E / E$ (the change rate of employment) and $\mathrm{dw} / \mathrm{w}$ (the change rate of marginal output of the monetized human resources), since we notice that the larger of the marginal contribution of $L$ the more willingness of hiring employees of companies. Figure 7 shows, the fluctuation of $\mathrm{d} w / w$ and $\mathrm{d} E / E$ are move to the same direction in shortterm, even though the range of fluctuation are different especially when the inflation is more serious.

The statistical data $Y$ and $r$ are the nominal values with money when calculate $\mathrm{d} w / w$. $E$ is the employed population. The nature of $E$ is the non-monetary real variable. 
When $\mathrm{d} P / P>0, \mathrm{~d} w / w$ which is calculated based on nominal values is greater than $\mathrm{d} E / E$ which is calculated based on real values. If Equation (10) is calculated based on real values $Y_{r}$ and $r_{r}$, then

$$
\frac{\mathrm{d} w_{r}}{w_{r}}=\frac{\mathrm{d} Y_{r}}{Y_{r}}-\frac{\alpha}{\beta} r_{r}
$$

Since $\mathrm{d} Y_{r} / Y_{r}=\mathrm{d} Y / Y-\mathrm{d} P / P$, and $r_{r}=r-\mathrm{d} P / P$, in which $\mathrm{d} P / P$ is the rate of inflation, then

$$
\frac{\mathrm{d} w_{r}}{w_{r}}=\frac{\mathrm{d} Y_{r}}{Y_{r}}-\frac{\alpha}{\beta} r_{r}=\left(\frac{\mathrm{d} Y}{Y}-\frac{\mathrm{d} P}{P}\right)-\frac{\alpha}{\beta}\left(r-\frac{\mathrm{d} P}{P}\right)=\left(\frac{\mathrm{d} Y}{Y}-\frac{\alpha}{\beta} r\right)-\left(1-\frac{\alpha}{\beta}\right) \frac{\mathrm{d} P}{P}
$$

Since $\mathrm{d} w / w=\mathrm{d} Y / Y-(\alpha / \beta) r$, the real variable $\mathrm{d} w_{r} / w_{r}$ and the nominal variable $\mathrm{d} w / w$ is expressed as

$$
\frac{\mathrm{d} w_{r}}{w_{r}}=\frac{\mathrm{d} w}{w}-\left(1-\frac{\alpha}{\beta}\right) \frac{\mathrm{d} P}{P}
$$

As Figure 8 shows, the fluctuation of $\mathrm{d}_{r} / w_{r}$ and $\mathrm{d} E / E$ are approximately similar. The reason for they are not completely consistent is because we assume $\alpha$ keep unchanged and therefore the change of $\alpha$ is not included in the impact on $\mathrm{d} w_{r} / w_{r}$. Above statistical data show that the change rate of employment $\mathrm{d} E / E$ is determined by the change rate of marginal real output of human resources $\mathrm{d} w_{r} / w_{r}$. Therefore we assume $\mathrm{d} E / E=\mathrm{d} w_{r} / w_{r}$ and replaced $\mathrm{d} w_{r} / w_{r}$ in Equation (11) with $\mathrm{d} E / E$, then

$$
\frac{\mathrm{d} E}{E}=\frac{\mathrm{d} Y_{r}}{Y_{r}}-\frac{\alpha}{\beta} r_{r}
$$

According to Equation (6) assume $\mathrm{d} Y_{r} / Y_{r} \approx r_{r}+\mathrm{d} r_{r} / r_{r}$, then

$$
\frac{\mathrm{d} E}{E}=\frac{\mathrm{d} Y_{r}}{Y_{r}}-\frac{\alpha}{\beta} r_{r}=r_{r}+\frac{\mathrm{d} r_{r}}{r_{r}}-\frac{\alpha}{\beta} r_{r}=\left(1-\frac{\alpha}{\beta}\right) r_{r}+\frac{\mathrm{d} r_{r}}{r_{r}}
$$

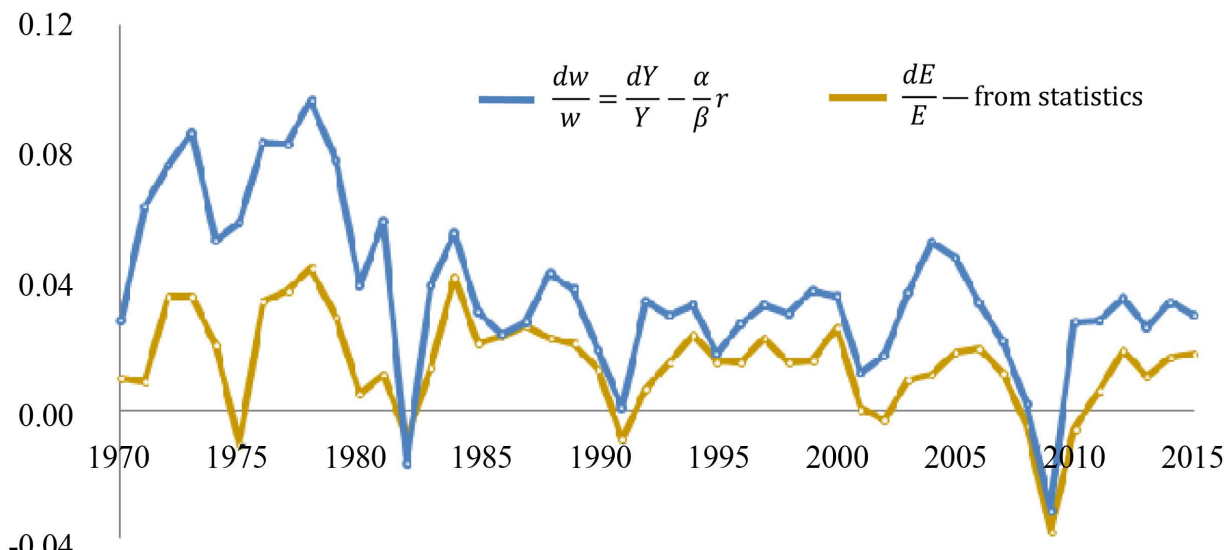

Figure 7. Relations between $\mathrm{d} w / w$ and $\mathrm{d} E / E$. Calculation and Resources: 1) $\mathrm{d} w / w=\mathrm{d} Y / Y-(\alpha / \beta) r$, in which $\alpha=1-\beta, \quad \beta$ is the compensation of employees in personal income. Data of wage income are from http:// www.bea.gov/. Data of $Y$ and $r$ see Figure 6. 2) $\mathrm{d} E / E \approx \Delta E / E$, data of employment population $E$ are from http://www.bls.gov/cps/cpsaat01.htm. 


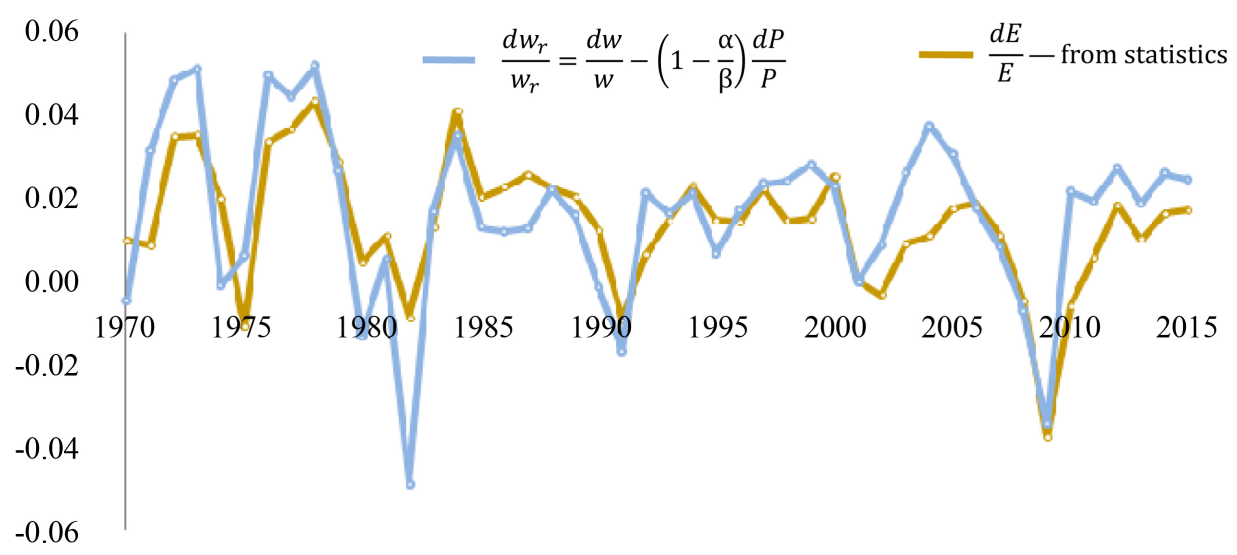

Figure 8. Relations between $\mathrm{d} w_{r} / w_{r}$ and $\mathrm{d} E / E$. Calculation and Resources: 1$)$ $\mathrm{d} w_{r} / w_{r}=\mathrm{d} w / w-(1-\alpha / \beta) \mathrm{d} P / P$. Data of $\mathrm{d} w / w, \alpha / \beta$, and $\mathrm{d} E / E$ see Figure 7. 2) $\mathrm{d} P / P \approx \Delta P / P, P$ is calculated based on the deflator of the nominal GDP and the real GDP. Data are from http://www.bea.gov.

The structure of Equation (13) is similar to that of $\mathrm{d} Y / Y=r+\mathrm{d} r / r$, therefore we can draw the phase diagram $r_{r} \sim \mathrm{d} E / E$ according to the phase diagram $r_{r} \sim \mathrm{d} r_{r} / r_{r}$ as Figure 4 shows. According to the rate of change of employed population $\mathrm{d} E / E$ and $R_{E}=E / N \quad$ (labor population as $N$, employed population as $E$ ), we can convert $\mathrm{d} E / E$ into $\mathrm{d} R_{E} / R_{E}: \frac{\mathrm{d} R_{E}}{R_{E}}=\frac{\mathrm{d}(E / N)}{E / N}=\frac{\mathrm{d} E}{E}-\frac{\mathrm{d} N}{N}$, according to Equation (13)

$$
\frac{\mathrm{d} R_{E}}{R_{E}}=\left(1-\frac{\alpha}{\beta}\right) r_{r}+\frac{\mathrm{d} r_{r}}{r_{r}}-\frac{\mathrm{d} N}{N}
$$

As Figure 9 shows, the direction of rotation and shape of the phase diagram $r_{r} \sim \mathrm{d} R_{E} / R_{E}$ is the same as that of $r_{r} \sim \mathrm{d} E / E$. Core variables $\overline{\mathrm{d} E / E}$ and $\overline{\mathrm{d} R_{E} / R_{E}}$ locate differently on the vertical coordinate, and the difference is determined by the change rate of labor population $\mathrm{d} N / N$. In other words, we get the phase diagram of $r_{r} \sim \mathrm{d} R_{E} / R_{E}$ by moving the phase diagram of $r_{r} \sim \mathrm{d} E / E$ downward a distance of $\mathrm{d} N / N$. Therefore, we can predict that the fluctuation of $\mathrm{d} E / E$ and $\mathrm{d} R_{E} / R_{E}$ are consistent.

According to Equation (13), the reason of short-term fluctuation of $\mathrm{d} E / E$ is the periodicity factor $\left(r_{r}+\mathrm{d} r_{r} / r_{r}\right)$, which is similar to the reason of the fluctuation of $\mathrm{d} Y / Y$. If there is no phase or only tiny difference between the fluctuation of $\mathrm{d} Y / Y$ and $\mathrm{d} Y_{r} / Y_{r}$, and the phase difference between $r$ and $r_{r}$ is small, then the fluctuating paths of $\mathrm{d} E / E, \mathrm{~d} R_{E} / R_{E}$ and $\mathrm{d} Y_{r} / Y_{r}$ would be consistent. The statistical data of Figure 10 can verify this conjecture.

The phase diagram $r_{r} \sim \mathrm{d} R_{E} / R_{E}$ in Figure 9 shows that the change rate of employment rate $\mathrm{d} R_{E} / R_{E}$ maybe 0 under the effect of $r_{r}$ sometimes. Mathematically, first order differential $\mathrm{d} R_{E}=0$ indicates there is extreme value or inflection point on $R_{E}$. Therefore, there is a phase diagram between $R_{E}$ and $\mathrm{d} R_{E} / R_{E}$ probably. The phase diagram $R_{E} \sim \mathrm{d} R_{E} / R_{E}$ in Figure 11 is from the statistical data. 


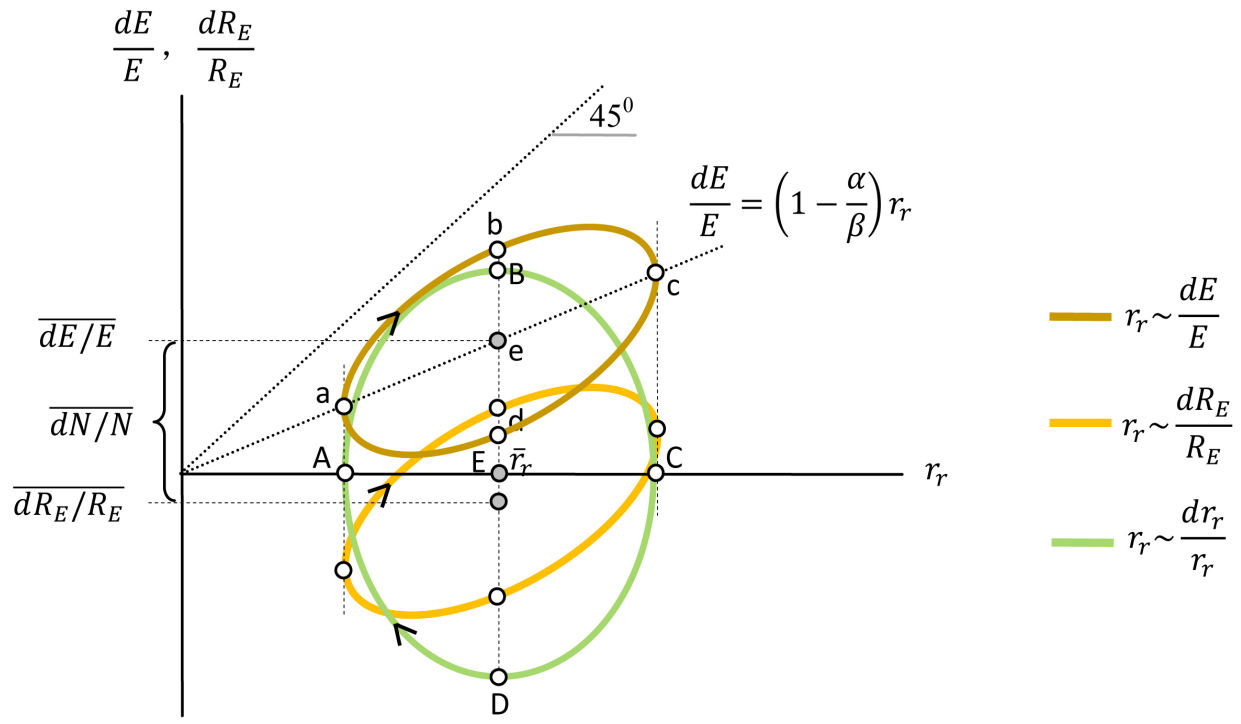

Figure 9. Relations between phase diagrams $r_{r} \sim \mathrm{d} E / E$ and $r_{r} \sim \mathrm{d} R_{E} / R_{E}$. Notice: Assume a phase diagram $r_{r} \sim \mathrm{d} r_{r} / r_{r}$, draw the auxiliary line $\mathrm{d} E / E=(1-\alpha / \beta) r_{r}$. Find points a, e, c corresponding to points $\mathrm{A}, \mathrm{E}, \mathrm{C}$ on $r_{r} \sim \mathrm{d} r_{r} / r_{r}$, fix other points according to the equation $\mathrm{d} E / E=(1-\alpha / \beta) r_{r}+\mathrm{d} r_{r} / r_{r}$. Then get the phase diagram $r_{r} \sim \mathrm{d} E / E$ by connecting these points.

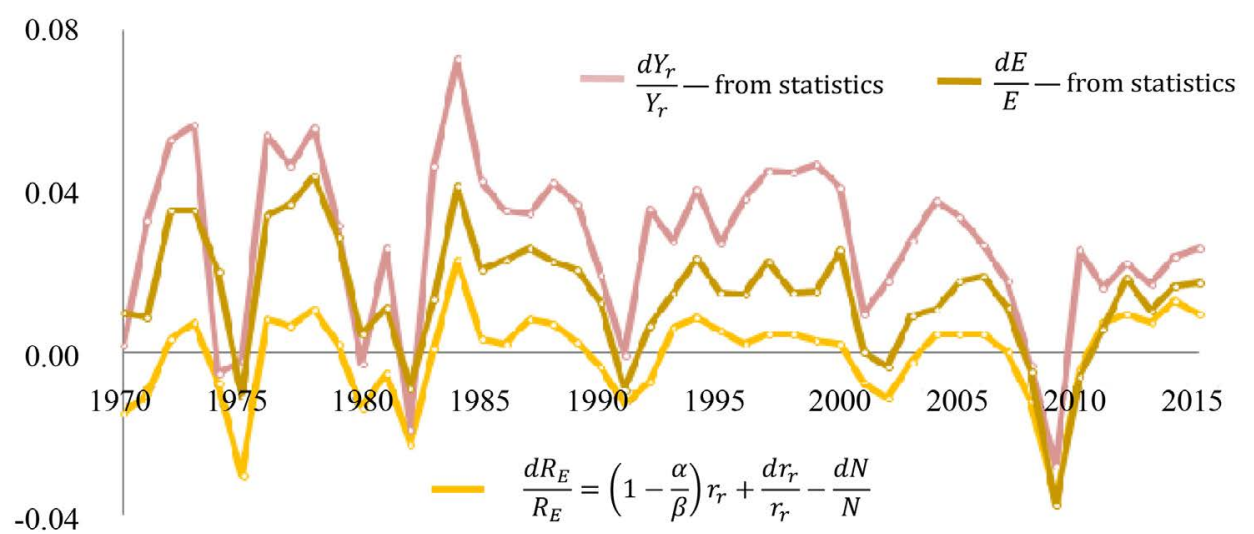

Figure 10. Relations between $\mathrm{d} E / E, \mathrm{~d} R_{E} / R_{E}$ and $\mathrm{d} Y_{r} / Y_{r}$. Calculation and resources: $\mathrm{d} Y_{r} / Y_{r}=\mathrm{d} Y / Y-\mathrm{d} P / P$, date of $Y$ is same Figure 6. Date of $P$ is same Figure 8.

$\mathrm{d} r_{r} / r_{r}=\mathrm{d} r / r-\mathrm{d} P / P, \mathrm{~d} r / r$ is same Figure 2. $\alpha / \beta$ is same Figure 7. Date of $N$ from http://www.bls.gov/cps/cpsaat01.htm.

In order to discuss the relationship between the unemployment rate $R_{U}$ and other macroeconomic variables based on the expression of Phillips curve, we often need to convert the employment rate $R_{E}$ to the unemployment rate $R_{U}$. Since the labor population $N$ is the sum of employed population $E$ and unemployed population $U$, namely $N=E+U$, then the relation between $R_{E}$ and $R_{U}$, and the relation between $\mathrm{d} R_{E} / R_{E}$ and $\mathrm{d} R_{U} / R_{U}$ are:

$$
R_{U}=\frac{U}{N}=\frac{N-E}{N}=1-\frac{E}{N}=1-R_{E}
$$



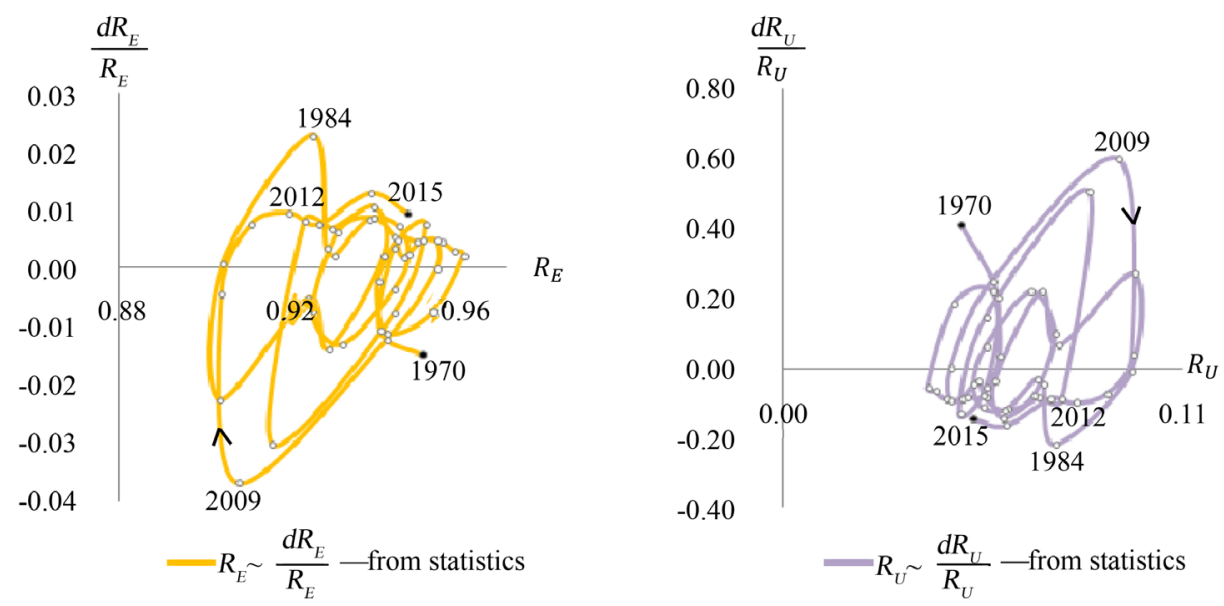

Figure 11. The phase diagram $R_{E} \sim \mathrm{d} R_{E} / R_{E}$ and $R_{U} \sim \mathrm{d} R_{U} / R_{U}$ in 1970-2015. Sources: Date of $R_{E}$ is same Figure 11. $R_{U}=1-R_{E}$.

$$
\frac{\mathrm{d} R_{U}}{R_{U}}=\frac{\mathrm{d}\left(1-R_{E}\right)}{1-R_{E}}=-\frac{\mathrm{d} R_{E}}{1-R_{E}}=-\frac{R_{E}}{1-R_{E}} \cdot \frac{\mathrm{d} R_{E}}{R_{E}}=\left(1-\frac{1}{R_{U}}\right) \frac{\mathrm{d} R_{E}}{R_{E}}
$$

Based on Equation (15) we can convert the phase diagram $R_{E} \sim \mathrm{d} R_{E} / R_{E}$ to $R_{U} \sim \mathrm{d} R_{U} / R_{U}$. The phase diagram of $R_{U} \sim \mathrm{d} R_{U} / R_{U}$ and $R_{E} \sim \mathrm{d} R_{E} / R_{E}$ is almost mirror images.

The $\mathrm{d} R_{U} / R_{U}$ is not the $R_{U}$. As there is a phase difference between $r_{r}$ and $\mathrm{d} r_{r} / r_{r}$, there is also a phase difference between $R_{U}$ and $\mathrm{d} R_{U} / R_{U}$, although their phase differences may not be significant in yearly statistical data. Figure 12 shows the fluctuation of $R_{U}$ and $\mathrm{d} R_{U} / R_{U}$ in the statistical data. Before 1986, the peaks of $R_{U}$ and $\mathrm{d} R_{U} / R_{U}$ appeared at the same time (which is the reason for inflation, which we will describe in later papers). After 1986, the peak of $R_{U}$ appeared later than $\mathrm{d} R_{U} / R_{U}$. The peaks of $\mathrm{d} R_{U} / R_{U}$ and $\mathrm{d} Y_{r} / Y_{r}$ are the same in Figure 10 , because $\mathrm{d} R_{U} / R_{U}$ and $\mathrm{d} R_{E} / R_{E}$ are the opposite of the peak, therefore the peak of $R_{U}$ will lag behind the peak of $\mathrm{d} Y_{r} / Y_{r}$ after 1986. This helps us understand the relationship and causes of fluctuations of unemployment and output.

While the above analysis explains the reasons for the cycle in the unemployment rate, there is one fundamental problem that remains unsolved: We cannot determine the value of $R_{U}$ or $\bar{R}_{U}$ according to Equations (17) and (14) in a business cycle. Because in the differential equation $\mathrm{d} R_{U} / R_{U}=\left(1-1 / R_{U}\right)\left[(1-\alpha / \beta) r_{r}+\mathrm{d} r_{r} / r_{r}-\mathrm{d} N / N\right]$, we need to know some initial conditions to determine $R_{U}$ or $\bar{R}_{U}$. As $Y_{r}$ and $\mathrm{d} Y_{r} / Y_{r}$, we cannot determine $Y_{r}$ from $\dot{Y}_{r}$ without knowing the initial condition of $Y_{r}$. This means that even if the $\mathrm{d} R_{U} / R_{U}$ of the two economic entities are exactly the same, their $R_{U}$ are not necessarily equal, because the marketization degree of employment and the culture of employment is different in different countries or regions.

Friedman considered although the unemployment state would be affected by "market imperfections, stochastic variability in demands and supplies, the cost of gathering information about job vacancies and labor availabilities, the costs of mobility, and so 


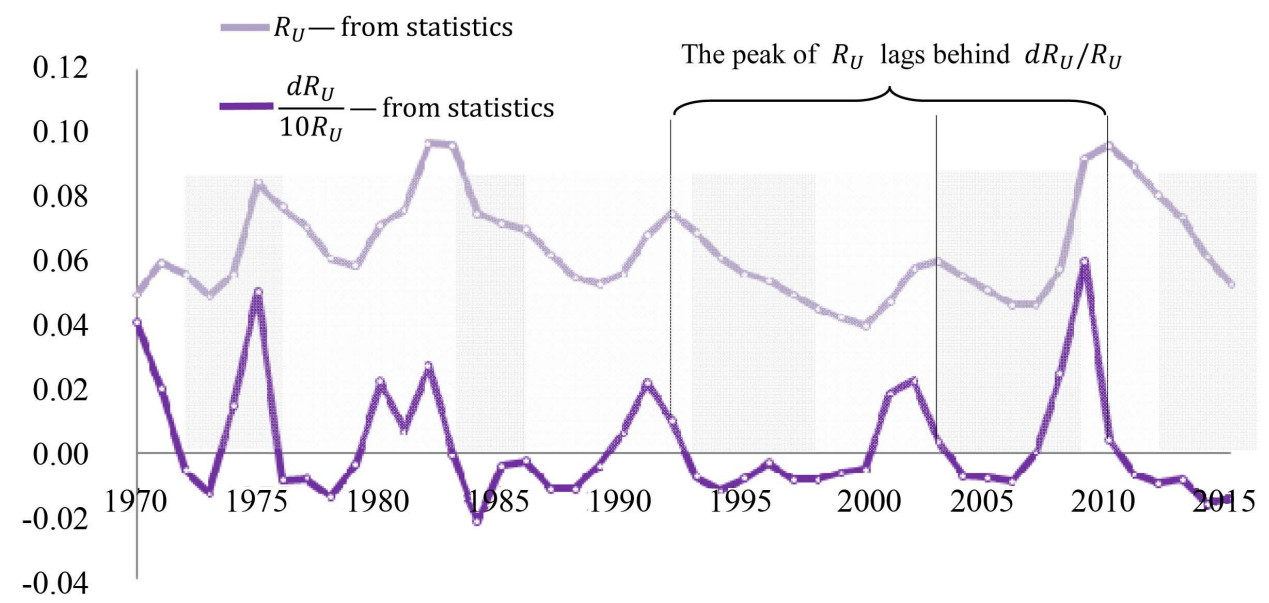

Figure 12. Relations between $R_{U}$ and $\mathrm{d} R_{U} / R_{U}$. Sources: The data is same Figure 11. For comparison purposes, $\mathrm{d} R_{U} / R_{U}$ is only $1 / 10$ of the original data.

on" [13], the unemployment rate fluctuated around the natural rate of unemployment in short term. Factors that Friedman took as examples are also initial conditions that affect the differential equation $R_{U}=f\left(\mathrm{~d} R_{U} / R_{U}\right)$. However, the natural rate of unemployment is not the core unemployment rate $\bar{R}_{U}$, since $\bar{R}_{U}$ move back and forwards in different cycles as Figure 11 shows.

\section{Conclusions}

\subsection{Hypothesis}

$\diamond$ Production function in the market system: $Y=A K^{\alpha} L^{\beta}$.

$\diamond$ A marginal condition: $r=\partial Y / \partial K=\alpha Y / K$.

$\diamond \mathrm{d} \alpha / \alpha=0, L^{\beta}=K^{\alpha}, \mathrm{d} E / E=\mathrm{d} w_{r} / w_{r}$.

\subsection{Results}

$\diamond$ The cycle equation about the output: $\mathrm{d} Y / Y=r+\mathrm{d} r / r$.

$\diamond$ The cycle equation about the employment: $\mathrm{d} R_{E} / R_{E}=(1-\alpha / \beta) r_{r}+\mathrm{d} r_{r} / r_{r}-\mathrm{d} N / N$, or $\mathrm{d} R_{U} / R_{U}=\left(1-1 / R_{U}\right)\left[(1-\alpha / \beta) r_{r}+\mathrm{d} r_{r} / r_{r}-\mathrm{d} N / N\right]$.

\subsection{Discussion}

$\diamond$ Traditional macroeconomics cannot logically explain contradictions of economic problems in long-term and short-term. Classical theory seems to be handy in explaining relationships between variables in long-term, but it is difficult to understand the phenomenon in short-term. Keynesian theory, while able to explain some phenomenon in short-term, but there will be ridiculous inference in long-term. From the model in this paper and "A kind of neither Keynesian nor neoclassical model (1): the fundamental equation" [12], we can logically and consistently see relationships between the macroeconomic variables in the long-term and the shortterm, and use statistical data to verify these relationships. 
$\diamond$ The cycle is affected by many factors, but as long as the marginal product of the economic system is not zero, there is the business cycle even without these external stochastic factors. Since $r$ and $\mathrm{d} r$ are the first and second order differentials of $Y$, the fluctuation of output is due the effect of the second order differential on the first order differential. Therefore the business cycle is fundamentally an endogenous producing action. Because both endogenous and exogenous forces have impacts on the cycle, so it is difficult to see two cycles are the same in terms of fluctuation and time consumption. On the interchangeability between inflation and unemployment in the Phillips Curve, we need to further our study on the relationship between money and Cobb-Douglas function.

$\diamond$ Due to the limited data sources and the heavy workload of processing data, this paper is limited to the verification of annual data. It is not known whether these periodic equations also apply to quarterly or monthly data.

\section{References}

[1] Kydland, F.E. and Prescott, E.C. (1988) The Workweek of Capital and Its Cyclical Implications. Journal of Monetary Economics, 21, 343-306. https://doi.org/10.1016/0304-3932(88)90035-9

[2] Kydland, F.E. and Prescott, E.C. (1990) Business Cycle: Real Facts and a Monetary Myth. Reserve Bank of Minneapolis Quarterly Review, 14, 3-18.

[3] Kydland, F.E. and Prescott, E.C. (1991) The Econometrics of the General Equilibrium Approach to Business Cycle. Scandinavian Journal of Economics, 93, 161-178. https://doi.org/10.2307/3440324

[4] Summers, L.H. (1986) Some Skeptical Observations on Real Business Cycle Theory. Federal Reserve Bank of Minneapolis Quarterly Review, 10, 23-27.

[5] Mankiw, N.G. (1989) Real Business Cycles: A New Keynesian Perspective. Journal of Economic Perspectives, 3, 79-90. https://doi.org/10.1257/jep.3.3.79

[6] Burside, C., Eichenbaum, M. and Rebelo, S. (1996) Sectoral Solow Residuals. European Economic Review, 40, 861-869. https://doi.org/10.1016/0014-2921(95)00095-X

[7] Basu, S. and Kimball, M.S. (1997) Cyclical Productivity with Unobserved Input Variation. NBER Working Paper Series, number 5915.

[8] Muellbauer, J. (1997) The Assessment: Business Cycles. Oxford Review of Economic Policy, 13, 1-18. https://doi.org/10.1093/oxrep/13.3.1

[9] Gall, J. (1999) Technology, Employment and Business Cycles: Do Technology Shocks Explain Aggregate Fluctuation? American Economic Review, 89, 249-271. https://doi.org/10.1257/aer.89.1.249

[10] Ramey, V.A. and Neville, F. (2002) Is the Technology-Driven Real Business Cycle Hypothesis Dead? Shocks and Aggregate Fluctuations Revisited. University of California at San Diego, Economics Working Paper Series qt6x80k3nx.

[11] Hayek, F.A. (1929) Monetary Theory and the Trade Cycle, New York: Augustus M. Kelley, 1966; reprint of 1933 English Edition, London: Jonathan Cape; originally published in German in 1929.

[12] Zhan, M.A. and Zhan, Z. (2016) A Kind of Neither Keynesian Nor Neoclassical Model (1): The Fundamental Equation. Open Access Library Journal, 3, e3207. https://doi.org/10.4236/oalib.1103207

[13] Friedman, M. (1968) The Role of Monetary Policy. American Economic Review, 58, 1-17. 
Submit or recommend next manuscript to OALib Journal and we will provide best service for you:

- Publication frequency: Monthly

- 9 subject areas of science, technology and medicine

- Fair and rigorous peer-review system

- Fast publication process

- Article promotion in various social networking sites (LinkedIn, Facebook, Twitter, etc.)

- Maximum dissemination of your research work

Submit Your Paper Online: Click Here to Submit

Or Contact service@oalib.com 